\title{
WILEY-VCH
}

\section{An Imperceptible Magnetic Skin}

Abdullah S. Almansouri *, Nouf A. Alsharif, Mohammed A. Khan, Liam Swanepoel, Altynay Kaidarova, Khaled N. Salama and Jurgen Kosel*

A. S. Almansouri, M. A. Khan, L. Swanepoel, A. Kaidarova, Prof. K. N. Salama and Prof. J. Kosel

Computer, Electrical, and Mathematical Sciences and Engineering Division (CEMSE) King Abdullah University of Science and Technology (KAUST)

Thuwal 23955, Saudi Arabia

E-mail: abdullah.almansouri@kaust.edu.sa

jurgen.kosel@kaust.edu.sa

A. S. Almansouri

Electrical Engineering and Computer Engineering, University of Jeddah, Dhahban 23881, Saudi Arabia.

\section{N. A. Alsharif}

Biological and Environment Science and Engineering, King Abdullah University of Science and Technology, Thuwal, Saudi Arabia.

Thuwal 23955, Saudi Arabia

Keywords: wearable electronics, composite magnet, eye tracking, flexible magnet, touchless control

Abstract - Flexible and wearable magnetoelectronics add intriguing new functionalities to our natural perception. Of particular interest regarding these artificial skins are wireless sensing and touchless interactions. Biocompatibility and imperceptibility are the most significant features of wearable devices attached to our bodies. In this work, a biocompatible magnetic skin is introduced. It offers extreme flexibility, stretchability $(>300 \%)$, and lightweight while maintaining a remanent magnetization up to $360 \mathrm{mT}$. The magnetic skin is comfortable to wear, can be realized in any desired shape or color, and adds tunable permanent magnetic properties to the surface it is applied to. It provides remote control functions and combined with magnetic sensors; it implements a complete wearable magnetic system. For example, eye tracking is realized by attaching the magnetic skin to the eyelid. The advantage that it does not require any wiring makes it an extremely viable solution for soft robotics and human-machine interactions. Wearing the magnetic skin on a finger or integrated 


\section{WILEY-VCH}

into a glove allows for remote gesture control. This type of application opens the door to new control concepts, relevant for people with disabilities, sterile environments, or the consumer industry. 


\section{WILEY-VCH}

The need for wearable electronics has increased significantly in the last two decades ${ }^{[1-3]}$. These electronics have a wide range of applications, including tracking the movement and activities of consumers, ${ }^{[1,2,4]}$ monitoring the health status of individuals, ${ }^{[5]}$ and serving as a human-to-machine interface. ${ }^{[6]}$ In fact, the global market of such devices is expected to reach $\$ 160$ billion by $2028 .{ }^{[7]}$ However, most commercially existing wearable electronics are in the form of smartwatches and fitness bands, as they are bulky and non-flexible. ${ }^{[3]}$ The key enabling features to attach wearable devices to the skin are biocompatibility, flexibility, light weight, comfort when wearing, and less visibility, in addition to providing accurate measurement and energy-efficient performance. ${ }^{[3,4,8]}$ Transducers are the central element in wearable electronics as they are mainly responsible for the performance, the placement of the device, the nature of the output signal, the complexity of the readout circuit and the overall power consumption. While many wearable and flexible sensors have been developed before, ${ }^{[1,}$

9] there is a surprising void when it comes to wearable actuators. Flexible magnetoelectronics is part of this rapidly progressing field of research, which has brought forward different types of flexible magnets ${ }^{[10,11]}$, sensors (such as flexible magnetic tunnel junctions, flexible magnetoimpedance sensors, and flexible hall sensors) ${ }^{[9,12]}$ and magnetic skins ${ }^{[1,13]}$. Mixing polydimethylsiloxane (PDMS, i.e., Sylgard 184) with magnetic powder is one of the most popular methods to achieve flexible magnets ${ }^{[1]}$. However, the stiffness of the Sylgard imposes limitations to the comfortable attachment and wearability ${ }^{[1]}$. In this work, we propose a biocompatible magnetic skin that is comfortable to wear and adds tunable permanent magnetic properties to a surface in any desired shape or color. The magnetic skin offers extreme flexibility and stretchability, whilst being light weight and maintaining a high remanent magnetization. The flexible properties of this material waive the sensation of its presence, when attached to the body, making it comfortable and imperceptible to wear. Such imperceptible magnetic skins enable gesture control in many different formats, 


\section{WILEY-VCH}

providing remote operation solutions for numerous applications. Here, we demonstrate the utilization of the magnetic skin for eye tracking and touchless control.

The ultra-flexible magnetic skin is made of a silicon-based elastomer matrix (Ecoflex, Smooth-on) and a permanent magnetic micro powder (NdFeB). Figure 1a-f illustrates the fabrication process. First, a mold is prepared with the desired shape and dimensions. After that, the composite is prepared by mixing the Ecoflec and the NdFeB powder. The mixture is then casted into the mold and planarized using a casting blade. After curing, the magnetic skin is magnetized in the out-of-plane direction. Before or after releasing the magnetic skin, it can be colored to match the skin tone of the user or any other preference by adding a thin layer of paint. This way, the magnetic skin can have a wide range of colors (Figure 1f).

The impact of the filler concentration on the modulus of elasticity (i.e., the flexibility of the material) and the remanent magnetization is illustrated in Figure 1g. Five different composite samples are prepared by mixing the $\mathrm{NdFeB}$ micropowder and Ecoflex in 1:2, 1:1, 2:1, 3:1, and 4:1 weight ratios. This corresponds to $\mathrm{NdFeB}$ weight concentrations of $33 \mathrm{wt} \%, 50 \mathrm{wt} \%$, $66 \mathrm{wt} \%, 75 \mathrm{wt} \%$, and $80 \mathrm{wt} \%$. While the $50 \mathrm{wt} \%$ composite is twice as rigid as the native elastomer, the $80 \%$ sample is 12.5 times more rigid than the native elastomer. Thus, the filler concentration has a deleterious effect on the flexibility of the composite, but it also has a beneficial impact on the magnetic properties of the composite. The coercivity of the composite is high $(0.56 \mathrm{~T} \text {, as for pure } \mathrm{NdFeB} \text { powder })^{[14]}$ and independent of the filler concentration. This avoids demagnetization of the composite in the presence of magnetic fields that may exist in the sensing environment (such as those in the vicinity of transformers, motors, etc.). The remanent magnetization of the $50 \mathrm{wt} \% \mathrm{NdFeB}$ composite is approximately one third of the $80 \mathrm{wt} \% \mathrm{NdFeB}$ composite. Thereby, going from $50 \mathrm{wt} \%$ to $80 \mathrm{wt} \% \mathrm{NdFeB}$ weight concentration increases the remanence by about $200 \%$, while increasing the rigidity by $540 \%$. Thus, it can be concluded that the $1: 1$ or $50 \mathrm{wt} \%$ NdFeB sample offers a good tradeoff 


\section{WILEY-VCH}

between flexibility and remanent magnetization, as shown later, and fits the needs for the eye tracking and touchless control. Moreover, the Young's modulus of the magnet skin with 50 wt $\% \mathrm{NdFeB}$ is more than 17 times lower than the Sylgard-based PDMS composite magnets, ${ }^{[11,15]}$ which is the most popular polymer matrix for flexible materials and magnets. The magnetic properties were tested over 1000 stress cycles (i.e., stretching and relaxing) with up to $80 \%$ strain. The measurement results show a constant magnetic stray field of the magnetic skin, confirming mechanical stability of the material. The detailed characterization and measurement results are presented in Supplementary Information-1

The magnetic skin is biocompatible, as was assessed using two methods: the PrestoBlue cell viability assay to show quantitative cell viability, and the LIVE/DEAD fluorescence staining method that uses calcein for live cells and ethidium homodimer-1 (EthD-1) for dead cells. The preparation methods of the samples are discussed in Supplementary Information2. The PrestoBlue assay result is presented in Figure 1h; it shows the biocompatibility of the magnetic skin by maintaining a high cell viability ( $>90 \%)$ when cultured for up to 3 days. In addition, the fluorescence staining method (Figure 1i, j) shows the ability of HCT 116 to grow in a confluent way on the magnetic skin. Most of the cells on the magnetic skin are calceinstained 72 hours after growth, indicating high biocompatibility similar to the control

(Figure 1i). Scanning electron microscopy (SEM) is employed to study the morphology of the cells on the magnetic skin. Figure $1 \mathrm{k}, 1$ show the ability of HCT116 cells to be elongated on the magnetic skin. Furthermore, they display a cell membrane rich in both filopodia and lamellipodia, and focal adhesion points similar to the control (Figure 1k).

\section{Non-invasive and comfortable tracking of blinking and eye movements utilizing the} magnetic skin, is illustrated in Figure 2a. The magnetic skin is directly attached to the eyelid, and a multi-axis magnetic sensor is located nearby. The latter can be affixed in different convenient locations, such as the frame of glasses, as an electronic tattoo attached to 


\section{WILEY-VCH}

the forehead, ${ }^{[16]}$ or integrated into a sleeping mask for tracking eye movements while sleeping. In such arrangements and due to the bulge structure of the cornea, any motion of the eye moves the magnetic skin, thus changing the magnetic field sensed by the multi-axis magnetic sensor. Such an implementation has vast applications for a wide range of consumers. ${ }^{[17]}$ For example, eye tracking is used as a human-computer interface especially for paralyzed people, ${ }^{[18,19]}$ in the gaming industry, ${ }^{[20]}$ to analyze individuals' sleep patterns ${ }^{[21]}$ to diagnose and wirelessly monitor some eye diseases such as ptosis of the eyelid (i.e., drooping of the eyelid), ${ }^{[22]}$ to observe the behavior of the eye in everyday life, ${ }^{[17]}$ and to monitor driver awareness. ${ }^{[23]}$ Nevertheless, existing methods are uncomfortable to wear, expensive, invasive, require wired connections or need the eyes to be wide open (conventional eye tracking methods are discussed in Supplementary Information-3) ${ }^{[18,24]} \mathrm{A}$ survey was conducted to evaluate the comfort level and the impact of having the magnetic skin attached to the eyelid (Supplementary Information-4). The survey consisted of 30 volunteers (10 females and 20 males aged from 17 to 36 ). With a confidence level exceeding $95 \%$ ( $p<0.05$, student's t-test), the discomfort level of attaching the magnetic skin onto the eyelid (including the physical and the emotional feelings) is below 1.2, with 0 meaning that the volunteer was not affected by the magnetic skin at all and 5 meaning it had a strong effect. In fact, the small percentage of the participant with discomfort level $\geq 2$ complained about the adhesive material (Vaseline ${ }^{\circledR}$ ) that was utilized to attach the magnetic skin to the eyelid, suggesting the use of another less viscous material could remedy this issue. Also, there is no clear difference (i.e., $\mathrm{p}>0.05$ ) between the comfort level perceived by males and females. This survey was regulated by the Saudi National Committee of Bio-Ethics (NCBE) and the Institutional Biosafety and Bioethics Committee (IBEC) at King Abdullah University of Science and Technology. 


\section{WILEY-VCH}

Tracking the eye movements is achieved by attaching a magnetic skin to the eyelid, as shown in Figure 2b. Figure $2 \mathrm{c}$ shows the measurement results regarding the movement of the eyeball in four cases: 1) looking upward and downward while the eyelid is open; 2) looking to the right and left while the eyelid is open; 3) looking upward and downward while the eyelid is closed; and 4) looking to the right and left while the eyelid is closed. Inspection of the data reveals that the magnetic field transverse to the forehead is sensitive to any eye movement. This is because the eyeball is not perfectly spherical: the cornea introduces a bulged surface. ${ }^{[25]}$ Upon the movement of the eyeball, the cornea pushes the eyelid; hence, the attached magnetic skin moves outward and inward. As a consequence, the transverse magnetic field varies. On the other hand, the magnetic field parallel to the forehead varies only when the user looks upward or downward (even when the eyelid is closed). In an ideal case, this should not change the parallel magnetic field, but moving the eyeball upwards and downwards results in moving the eyelid upwards and downwards too. Therefore, the attached magnetic skin moves and changes the parallel magnetic field. In other words, changes in both the parallel and the transverse magnetic fields imply supraversion/infraversion behavior of the eye, while changes in the transverse magnetic field only imply levoversion/supraversion.

Touchless control utilizing the magnetic skin, is realized by attaching the magnetic skin to the hand, allowing the user to control devices by hovering above a touchless control element, which could be key, switch, pad etc., and without physically touching it. This is especially relevant in laboratories or medical practices, where contamination is of concern. Existing techniques, such as physical buttons are susceptible to contaminations, and voicebased interfaces usually cannot distinguish between different people speaking in the same room, besides being relatively expensive. Thermal or capacitive techniques are subject to accidental activation, when any part of anybody is in proximity to the sensor. Body-worn sensors like accelerometers and gyroscopes cannot provide the exact trajectory in addition to 


\section{WILEY-VCH}

requirement of wearing extra devices. Other proximity sensing techniques usually require computers to analyze the gesture and the position of the hand, which adds to the complexity and the cost of the system, and they are vulnerable to accidental activations. ${ }^{[26]}$ For such applications, the magnetic skin can be utilized for touchless control. It can be comfortably worn on the hand (Figure 3a, b) with the ability to match its color to the skin tone (Figure 3c). Also, it is possible to integrate the magnetic skin into a glove (Figure 3d). The extreme elasticity of the magnetic skin masks its presents and maintains the original flexibility of the glove (Figure 3e, f and Supplementary Movie 1). Virtual control keys were realized using hidden magnetic sensors (Supplementary Information-5). This way, the desired key can be activated only, when the tip of the glove with the magnetic skin is within a pre-determined distance (threshold distance) from the magnetic skin. Note that, this controlling key cannot be activated unless the magnet is within the threshold distance; hence, accidentally pressing or hovering above the controlling keys with any other part of the body or using other nonmagnetized objects is eliminated (Supplementary Movie 2). Figure 3g-h shows the activation of the touchless control by hovering over a hidden magnetic sensor with a magnetic skin integrated into the fingertip of a glove.

In summary, the imperceptible magnetic skin is biocompatible and highly flexible and stretchable. The viability of cells growing on the magnetic skin remains very high, as evaluated using the PrestoBlue cell viability assay and the LIVE /DEAD fluorescence staining method. It is up to 17 times more flexible than the more popular Sylgard-based PDMS composites. Combining the features of flexibility, stretchability, and biocompatibility, along with versatility in shape and color, makes the magnetic skin imperceptible to wear. Thus, it can be comfortably attached to relatively sensitive areas, such as the eyelid, as confirmed by a survey including 30 volunteers. In this case, a nearby multi-axis magnetic sensor can be conveniently integrated into eyeglasses to wirelessly track the movement of the eyeball or the 


\section{WILEY-VCH}

blink of the eye. Furthermore, a touchless control switch is realized by attaching the magnetic skin to the fingertip of a glove. This method eliminates accidental activation and contamination of the control keys, meanwhile, the extreme flexibility of the magnetic skin maintains the elasticity of the glove. Ultimately, the magnetic skin can be combined with flexible and stretchable magnetic sensors on the same substrate, where many different kinds have been realized on polymer substrates before, except for tunnel magnetoresistance sensors, to provide combined remote sensing and actuation.

\section{Experimental Section}

The magnetic skin is realized using Ecoflex 00-50 (Smooth-on) and MQP-16-7FP NdFeB permanent magnetic micro powder. The composite is prepared by mixing $25 \mathrm{wt} \%$ Ecoflex part A, $25 \mathrm{wt} \%$ Ecoflex part B, and $50 \mathrm{wt} \% \mathrm{NdFeB}$ powder. After mixing the composite for about 3 minutes, vacuum desiccation is performed for 10 minutes to eliminate air bubbles, which can adversely impact the mechanical and magnetic properties of the composite. The mixture is then casted into a polylactic acid (tough PLA, Ultimaker) 3D printed mold with the desired configuration, and subsequently cured at room temperature for 3 hours. The magnetic skin is then magnetized using 1.8 T external magnetic field. Finally, coloring the magnetic skin is achieved using Psycho Paint (Smooth-on) mixed with the appropriate color pigments of Slic Pic (Smooth-on), and leaving it to dry for 2 hours.

Tracking the eye movements is achieved by attaching a $10 \times 2 \times 0.7 \mathrm{~mm}^{3}$ magnetic skin weighing $19 \mathrm{mg}$ to the eyelid (about 1-2 mm above the eyelashes, as shown in Figure $2 \mathrm{~b}$ ). A small commercially available development board (STMicroelectronics SensorTile, STEVALSTLCS01V1) that offers a multi-axis magnetic sensor and a Bluetooth module for wireless communication is attached to the frame of the glasses. The sensor is placed about $15 \mathrm{~mm}$ away from the magnetic skin. 


\section{WILEY-VCH}

\section{Supporting Information}

Supporting Information is available from the Wiley Online Library.

\section{Acknowledgements}

Figure 2a was created by Heno Hwang, scientific illustrator at King Abdullah University of Science and Technology (KAUST).

Received: ((will be filled in by the editorial staff))

Revised: ((will be filled in by the editorial staff)) Published online: ((will be filled in by the editorial staff))

\section{References}

[1] Bermúdez, G. S. C.; Karnaushenko, D. D.; Karnaushenko, D.; Lebanov, A.; Bischoff, L.; Kaltenbrunner, M.; Fassbender, J.; Schmidt, O. G.; Makarov, D., Science advances 2018, $4(1)$, eaao2623.

[2] Jeong, J. W.; Yeo, W. H.; Akhtar, A.; Norton, J. J.; Kwack, Y. J.; Li, S.; Jung, S. Y.;

Su, Y.; Lee, W.; Xia, J., Advanced Materials 2013, 25 (47), 6839-6846.

[3] Servati, A.; Zou, L.; Wang, Z. J.; Ko, F.; Servati, P., Sensors 2017, 17 (7), 1622.

[4] Stoppa, M.; Chiolerio, A., sensors 2014, 14 (7), 11957-11992.

[5] a) Lee, H.; Kim, E.; Lee, Y.; Kim, H.; Lee, J.; Kim, M.; Yoo, H.-J.; Yoo, S., Science advances 2018, 4 (11), eaas9530; b) Löfhede, J.; Seoane, F.; Thordstein, M., Sensors 2012, 12 (12), 16907-16919; c) Coosemans, J.; Hermans, B.; Puers, R., Sensors and Actuators A: Physical 2006, 130, 48-53.

[6] a) Liu, Y.; Norton, J. J.; Qazi, R.; Zou, Z.; Ammann, K. R.; Liu, H.; Yan, L.; Tran, P. L.; Jang, K.-I.; Lee, J. W., Science advances 2016, 2 (11), e1601185; b) Liao, L.-D.; Chen, C.-Y.; Wang, I.-J.; Chen, S.-F.; Li, S.-Y.; Chen, B.-W.; Chang, J.-Y.; Lin, C.-T., Journal of neuroengineering and rehabilitation 2012, 9 (1), 5. 


\section{WILEY-VCH}

[7] Hayward, J. Wearable Sensors 2018-2028: Technologies, Markets and Players. https://www.idtechex.com/research/reports/wearable-sensors-2018-2028-technologiesmarkets-and-players-000555.asp (accessed 25 November).

[8] Pang, C.; Lee, C.; Suh, K. Y., Journal of Applied Polymer Science 2013, 130 (3), $1429-1441$.

[9] a) Amara, S.; Sevilla, G.; Torres, A.; Hawsawi, M.; Mashraei, Y.; Mohammed, H.;

Cruz, M. E.; Ivanov, Y.; Jaiswal, S.; Jakob, G., arXiv preprint arXiv:1804.01298 2018; b) Li, B.; Kavaldzhiev, M. N.; Kosel, J., Journal of Magnetism and Magnetic Materials 2015, 378, 499-505.

[10] a) Alfadhel, A.; Li, B.; Zaher, A.; Yassine, O.; Kosel, J., Lab on a Chip 2014, 14 (22), 4362-4369; b) Khan, M. A.; Alfadhel, A.; Kosel, J., IEEE Transactions on Magnetics 2016, 52 (7), 1-4; Alfadhel, A.; Kosel, J., Advanced Materials 2015, 27 (47), 7888-7892; c)

Alfadhel, A.; Khan, M. A.; de Freitas, S. C.; Kosel, J., IEEE Sensors Journal 2016, 16 (24), 8700-8705.

[11] Kaidarova, A.; Khan, M. A.; Amara, S.; Geraldi, N. R.; Karimi, M. A.; Shamim, A.;

Wilson, R. P.; Duarte, C. M.; Kosel, J., Advanced Engineering Materials 2018.

[12] Wang, Z.; Shaygan, M.; Otto, M.; Schall, D.; Neumaier, D., Nanoscale 2016, 8 (14), $7683-7687$

[13] Bermúdez, G. S. C.; Fuchs, H.; Bischoff, L.; Fassbender, J.; Makarov, D., Nature Electronics 2018, 1 (11), 589.

[14] Magnequench MQP-S-11-9-20001-070 Isotropic Powder. https://mqitechnology.com/wp-content/uploads/2017/09/mqp-16-7-20068-070.pdf.

[15] a) Wang, W.; Yao, Z.; Chen, J. C.; Fang, J., Journal of Micromechanics and Microengineering 2004, 14 (10), 1321; b) Khan, M. A.; Mohammed, H.; Kosel, J., Advanced Engineering Materials 2018, 20 (11), 1800492. 


\section{WILEY-VCH}

[16] Kabiri Ameri, S.; Ho, R.; Jang, H.; Tao, L.; Wang, Y.; Wang, L.; Schnyer, D. M.; Akinwande, D.; Lu, N., ACS nano 2017, 11 (8), 7634-7641.

[17] Skotte, J.; Nøjgaard, J.; Jørgensen, L.; Christensen, K.; Sjøgaard, G., European journal of applied physiology 2007, 99 (2), 113-119.

[18] Duchowski, A. T., Theory and practice 2007, 328.

[19] Horowitz, B. T. Eye-tracking technology allows paralyzed people to control PCs, tablets. https://blog.dell.com/en-us/eye-tracking-technology-allows-paralyzed-people-tocontrol-pcs-tablets/ (accessed February 14).

[20] Corcoran, P. M.; Nanu, F.; Petrescu, S.; Bigioi, P., IEEE Transactions on Consumer Electronics 2012, $58(2)$.

[21] Takahashi, K.; Atsumi, Y., Sleep 1997, 20 (9), 743-752.

[22] a) Frueh, B. R., Ophthalmology 1980, 87 (10), 1019-1021; b) Azri, M.; Young, S.; Lin, H.; Tan, C.; Yang, Z. In Diagnosis of Ocular Myasthenia Gravis by means of tracking eye parameters, Engineering in Medicine and Biology Society (EMBC), 2014 36th Annual International Conference of the IEEE, IEEE: 2014; pp 1460-1464.

[23] Smith, P.; Shah, M.; da Vitoria Lobo, N. In Monitoring head/eye motion for driver alertness with one camera, icpr, IEEE: 2000; 4636.

[24] a) Schwarz, J. S.; Sridharan, D.; Knudsen, E. I., Frontiers in systems neuroscience 2013, 7, 91; b) Heide, W.; Koenig, E.; Trillenberg, P.; Kömpf, D.; Zee, D., Electroencephalogr Clin Neurophysiol Suppl 1999, 52, 223-240.

[25] Gray, H.; Standring, S., Gray's anatomy. Arcturus Publishing: 2008.

[26] Hettig, J.; Saalfeld, P.; Luz, M.; Becker, M.; Skalej, M.; Hansen, C., International journal of computer assisted radiology and surgery 2017, 12 (9), 1643-1653. 


\section{WILEY-VCH}

a

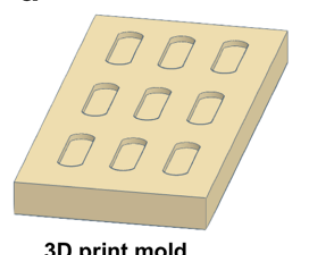

3D print mold
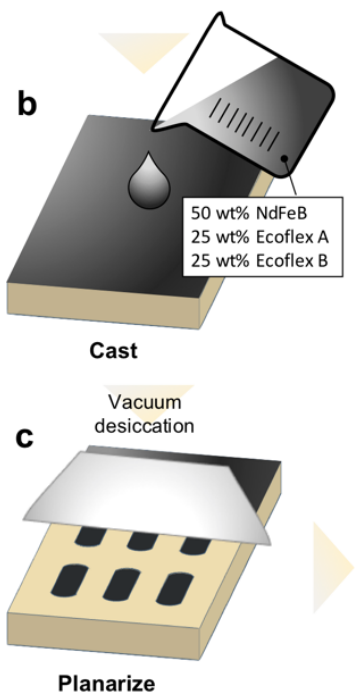

f

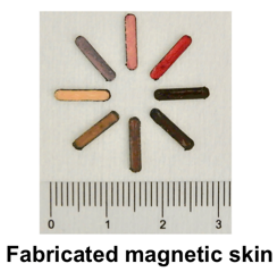

e

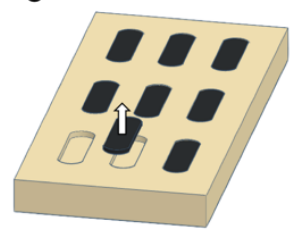

Release

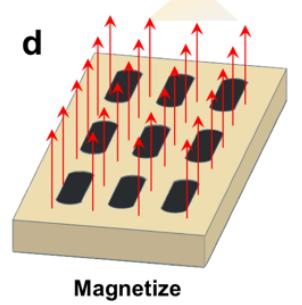

g

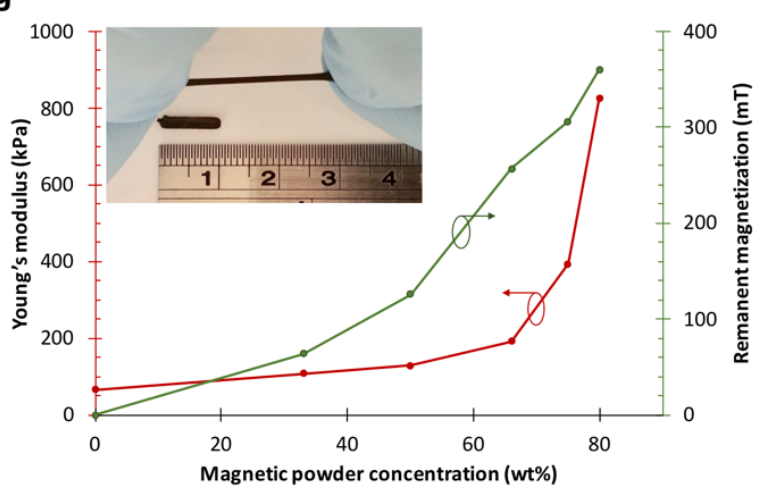

h

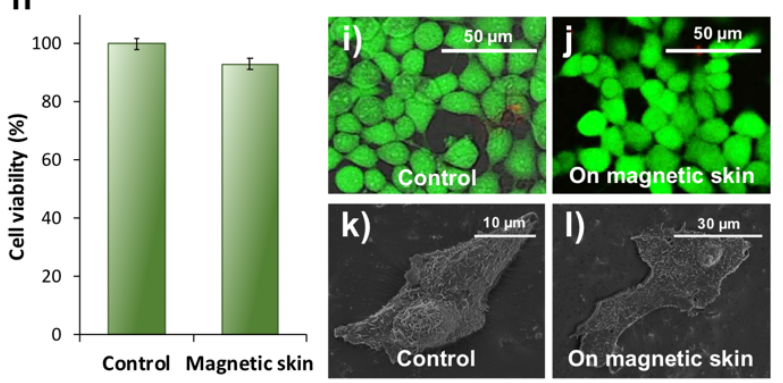

Figure 1. The magnetic skin is achieved by: a) 3D printing a mold with the desired shape, b) preparing the mixture of the magnetic skin by mixing the silicon-based elastomer matrix with the magnetic powder, c) planarizing the mixture into the mold, d) magnetizing the magnetic skin by applying an external magnetic field of $1.8 \mathrm{~T}$, and finally e) releasing the magnetic skin. f) Samples of the magnetic skin with a wide range of color options. g) Physical characterization of the modulus of elasticity and remanent magnetizations versus different concentration of the magnetic powered incorporated in the magnetic skin, where increasing the filler concentration results in a stiffer material with higher remanent magnetization. The inset shows a $50 \mathrm{wt} \%$ magnetic skin with more than $300 \%$ stretchability. $h$ to l) Biocompatibility assessment of the magnetic skin. h) PrestoBlue assay showing the viability of HCT116 cells grown on top of the magnetic skin after three days. The control shows the viability of cells growing on a cover slide. Error bars represent the standard deviation of six replicates. i to l) Confocal images of calcein (green-live cells)/EthD-1 (red-dead cells) stained HCT 116 cells grown on top of the magnetic skin. i) The positive control cells grown on top of a cover slide. j) cells on top of the magnetic skin. k) A positive control of an HCT 116 cell grown on a cover slide. 1) ESM image of HCT116 cell grown on the magnetic skin showing a flattened surface with cellular protrusions and focal adhesion points similar to the control. 


\section{WILEY-VCH}

a
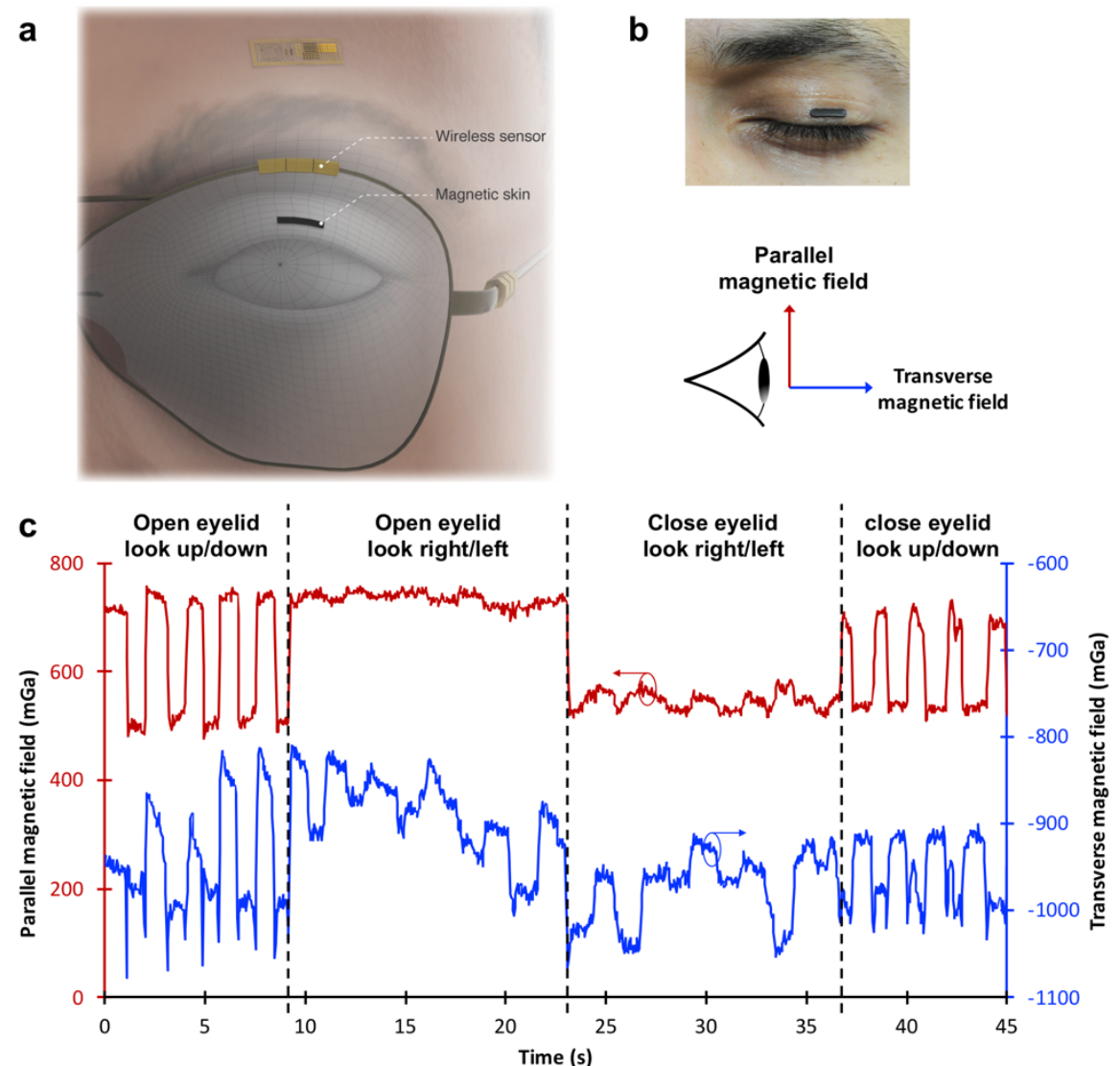

b
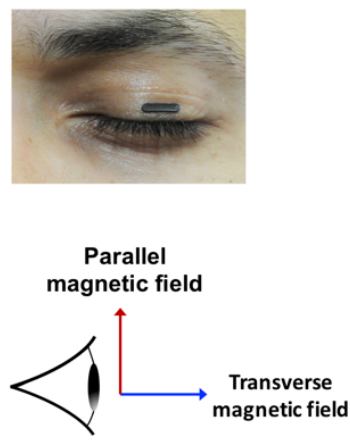

close eyelid clid

$\begin{array}{lll}\text { look right/left } & \text { look up/down }\end{array}$

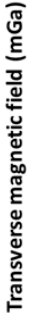

Figure 2. a) A magnetic skin attached to the eyelid to track eye movements. The movement of the eye results in varying the magnetic field that is detected with a nearby multi-axis magnetic sensor. This magnetic sensor can be in different locations, for example on the frame of the glasses or directly attached to the body. b) The location of the magnetic skin on the eyelid, where the transverse magnetic field is perpendicular to the facial plane, and the parallel magnetic field is longitudinal to the facial plane. c) The measured magnetic field at a nearby multi-axis magnetic sensor attached to the frame of the glasses. Looking upward or downward results in changing both the parallel and the transverse magnetic fields. On the other hand, looking to the right or the left results in changing the transverse magnetic field only. 


\section{WILEY-VCH}
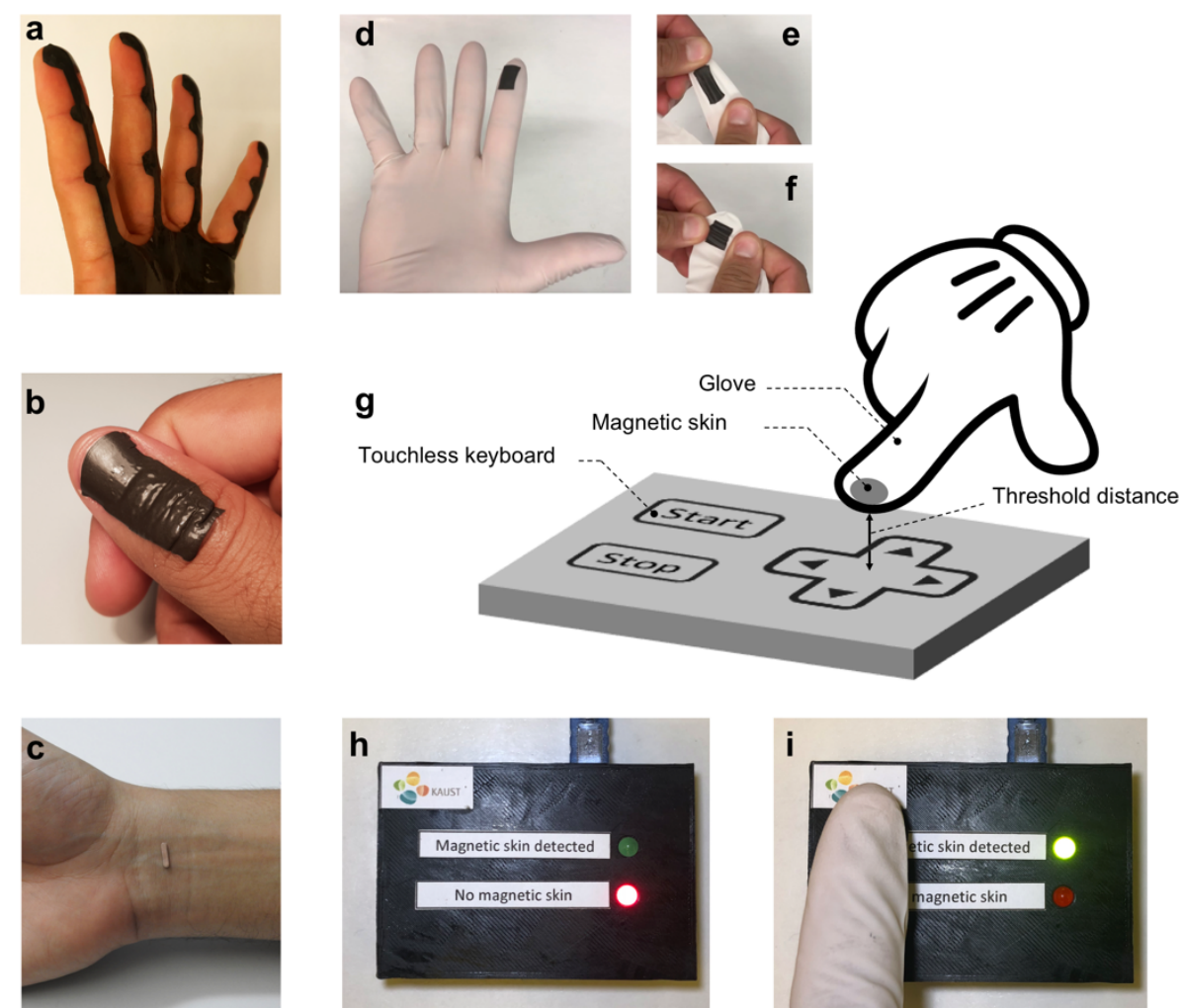

Figure 3. $a$ and b) A magnetic skin conforming to the natural topography of the hand and the thumb, respectively. c) The magnetic skin attached to the arm with a color matching the skin tone of the user, $d$ to $f$ ) The magnetic skin is attached to the tip of a glove, maintaining the flexibility and searchability of the glove. g) The integration of the magnetic skin into a glove is utilized as a touchless control to eliminate contamination and accidental activation of the buttons. To activate a key, the magnetic skin should be located within a pre-determined threshold distance. $h$ and i) The presence of the magnetic skin within the threshold distance activates the touchless control and turns on the green LED. 


\section{WILEY-VCH}

An ultra-flexible magnetic skin is proposed. It is biocompatible, flexible, stretchable and can take on any shape or color, making it imperceptible when worn. The versatility of the magnetic skin is demonstrated by creating magnetic eyes, fingers and hands. The magnetic skin is a wearable passive element; hence, it is a viable solution for soft robotics and human-machine interactions.

Keywords wearable electronics, composite magnet, eye tracking, flexible magnet, touchless control

A.S. Almansouri ${ }^{*}$ N. A. Alsharif, M. A. Khan, L. Swanepoel, A. Kaidarova, K. N. Salama and J. Kosel*

\section{An Imperceptible Magnetic Skin}

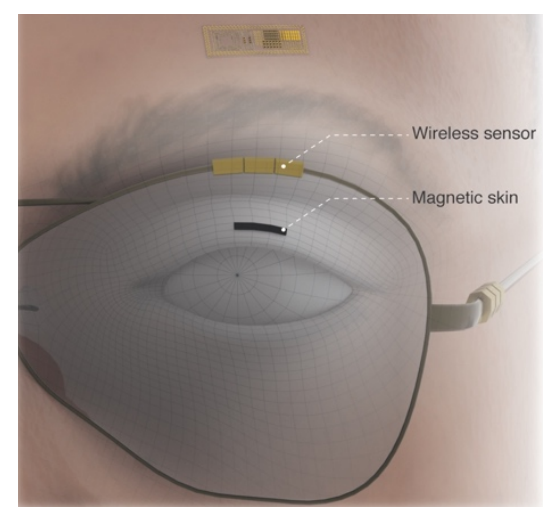


Copyright WILEY-VCH Verlag GmbH \& Co. KGaA, 69469 Weinheim, Germany, 2018.

Supporting Information

\section{An Imperceptible Magnetic Skin}

Abdullah S. Almansouri * Nouf A. Alsharif, Mohammed A. Khan, Liam Swanepoel, Altynay Kaidarova, Khaled N. Salama and Jurgen Kosel

\section{Supplementary Information-1: Physical characterization of the magnetic skin}
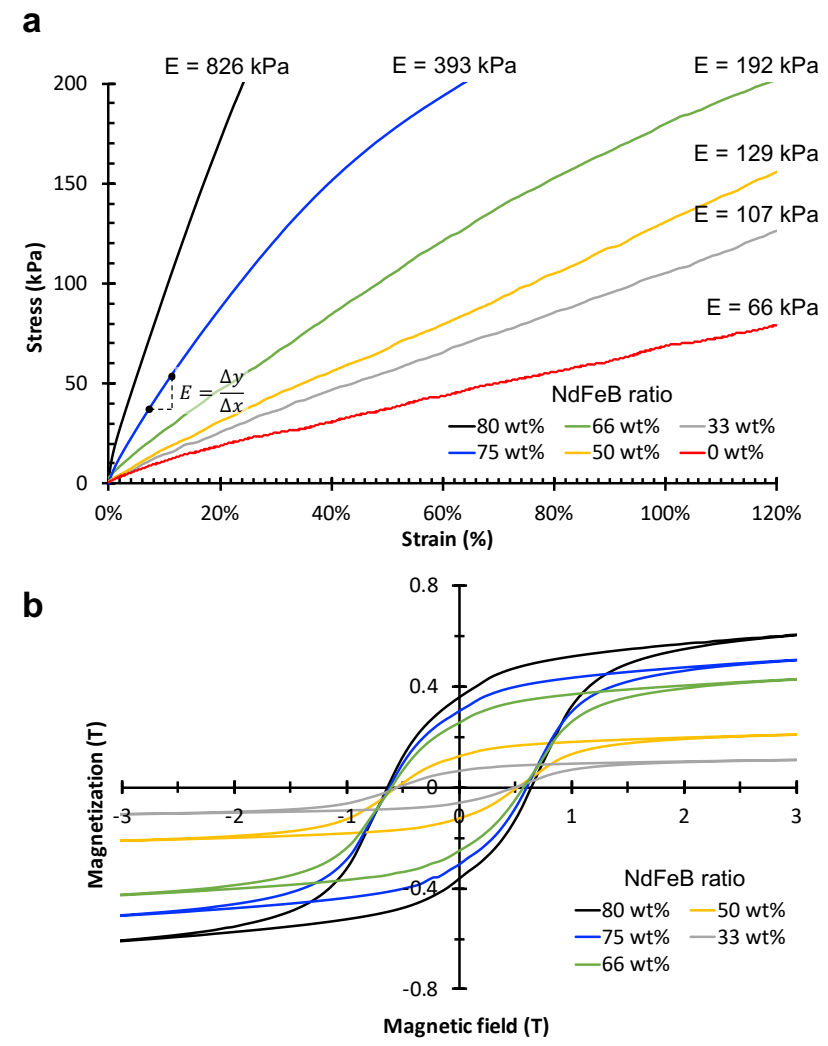

Figure S1. Characterization of the magnetic skin for different concentrations of the magnetic powder. a) shows the stress-strain curves of the magnetic skin, where increasing the filler concentration results in a stiffer material. b) shows the magnetization curves of the magnetic skin, where increasing the filler concentration results in higher remanent and saturation 


\section{WILEY-VCH}

magnetizations. Also, the coercivity of the composite is high and independent of the filler concentration. 

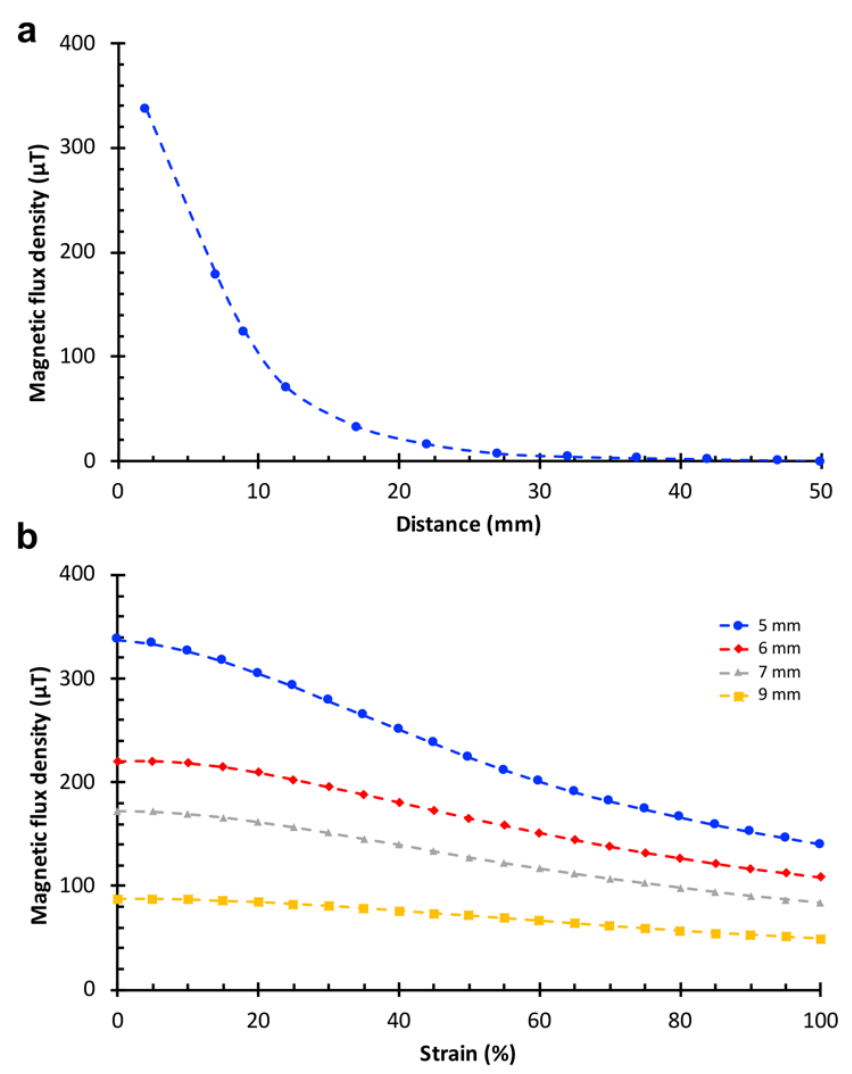

Figure S2. The measured stray magnetic field of a $10 \times 2 \times 0.7 \mathrm{~mm}^{3}$ magnetic skin sample, where the magnetization is out of plane (along the $0.7 \mathrm{~mm}$ axis). (a) Shows the magnetic field as a function of distance between the sample and the magnetic sensor. The measurement results show a reduction in the magnetic field with increased distance. (b) Shows the magnetic field as a function of strain. Note, the strain is along the $10 \mathrm{~mm}$ axis. Stretching the magnetic skin results is less magnetic particles per unit length by thinning the sample, hence, the magnetic field decreases accordingly.

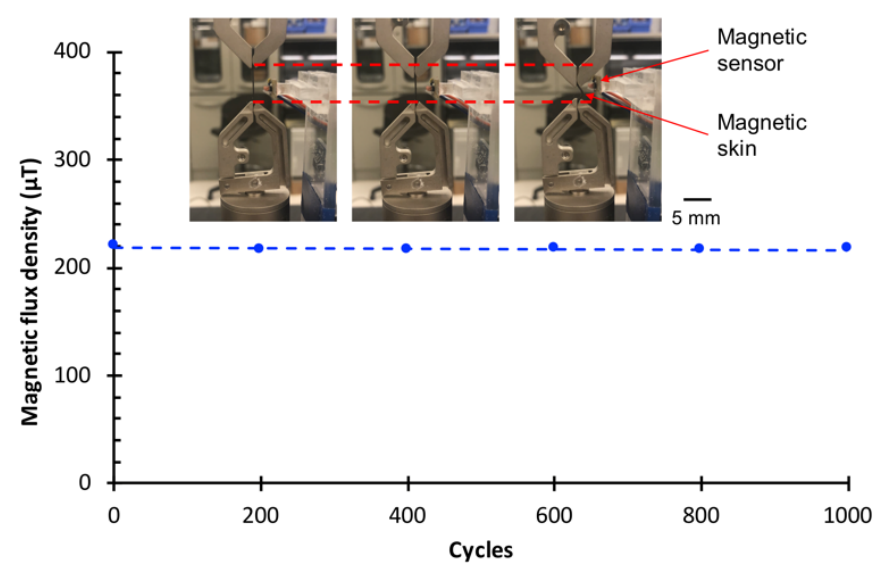




\section{WILEY-VCH}

Figure S3. Magnetic field as a function of stress cycles. Each cycle consisted of stretching and relaxing a $10 \times 2 \times 0.7 \mathrm{~mm}^{3}$ magnetic skin sample. A magnetic sensor was placed $6 \mathrm{~mm}$ away from the sample and shows a stable measurement of $217 \mu \mathrm{T}$. The insets shows the measurement setup and images of one stress cycle. 


\section{WILEY-VCH}

\section{Supplementary Information -2: Biocompatibility}

a. Cell culture

HCT 116 (ATCC CCL247) cells were cultured in McCoy's medium 5A 1× with 1glutamine with $10 \%$ fetal bovine serum and $1 \%$ penicillin-streptomycin. To detach and count the cells, StemPro Accutase and trypan blue were used, respectively. The cells were grown inside of a $37^{\circ} \mathrm{C}$, humidified incubator with $5 \% \mathrm{CO}_{2}$. All of the supplements and the media were bought from Gibco, Life Technologies.

For the cell viability assay and TEM images, the magnetic skin was placed in 96-well or 6well plates and washed three times with ethanol followed by three times with $0.01 \mathrm{M}$ PBS. After that, the surface was coated with Fibronectin bovine plasma $(10 \mu \mathrm{g} / \mathrm{ml})$ for $1 \mathrm{~h}$ to enhance the attachment of the cells. Finally, 500,000 cells were seeded on top of the magnetic skin for three days.

\section{b. Cell viability}

Two methods were used to study the biocompatibility of the magnetic skin. (1) The first was the LIVE/DEAD ${ }^{\circledR}$ Viability/Cytotoxicity Kit (Molecular Probes ${ }^{\mathrm{TM}}$; Eugene Oregon, USA). Based on the company's protocol, the cells were stained using a $2 \mu \mathrm{M}$ calcein AM and $4 \mu \mathrm{M}$ ethidium homodimer-1 (EthD-1) in PBS for $20 \mathrm{~min}$ at room temperature. Then, they were washed with $0.01 \mathrm{MPBS}$ and analyzed by confocal microscopy. (2) The second was the PrestoBlue cell viability assay (Cat. No. A13261). Based on the vendor's protocol, after incubating the cells in 96-well plate for three days, the culture medium was carefully removed and exchanged with a fresh one $(90 \mu 1)$. Subsequently, $10 \mu 1$ PrestoBlue reagent was added directly to the well and incubated for $2 \mathrm{~h}$ at $37^{\circ} \mathrm{C}$. To analyze the data, the absorbance microplate reader was used at $570 \mathrm{~nm}$. The experiment was repeated three times with nearly identical results. 


\section{WILEY-VCH}

\section{c. Preparation for scanning electron microscopy (SEM) imaging}

After three days of incubating HCT116 cells with magnetic skin, the cells were washed with $1 \mathrm{X}$ PBS and then fixed in $2.5 \%$ glutaraldehyde in $0.1 \mathrm{M}$ PBS $4^{\circ} \mathrm{C}$ overnight. After fixation, cells were washed with $0.1 \mathrm{M}$ PBS buffer three times for $15 \mathrm{~min}$ per wash. The cells were then post-fixed in $1 \%$ osmium tetroxide in $0.1 \mathrm{M}$ PBS for $1 \mathrm{~h}$ in the dark, after which they were washed with deionized water three times for 15 min per wash. This was followed by a serial dehydration using ethanol at $10 \%, 30 \%, 50 \%, 70 \%, 90 \%$, and $100 \%$ for 5 min each.

For drying, the sample was left in a 1:2 solution of Hexamethyldisilazane:100\% ethanol for $20 \mathrm{~min}$, a 2:1 solution of Hexamethyldisilazane:100\% ethanol for $20 \mathrm{~min}$, and $100 \%$ Hexamethyldisilazane for 20 minutes. Then it was kept open in fresh $100 \%$ Hexamethyldisilazane inside the fume hood overnight. The next day, the sample was coated with $5 \mathrm{~nm}$ of platinum-palladium before SEM imaging. 


\section{WILEY-VCH}

\section{Supplementary Information-3: Conventional methods for tracking eye movement}

Tracking blinking and eye movements has widespread applications and is becoming increasingly interesting. ${ }^{[1]}$ For example, it has great potential as a human-computer interface ${ }^{[2,3]}$ especially for paralyzed people, ${ }^{[4]}$ in the gaming industry, ${ }^{[5]}$ to analyze individuals' sleep patterns, ${ }^{[6]}$ to diagnose and wirelessly monitor some eye diseases such as ptosis of the eyelid (i.e., drooping of the eyelid), ${ }^{[7]}$ to observe the behavior of the eye in everyday life, ${ }^{[1]}$ and to monitor driver awareness. ${ }^{[8]}$ The most popular methods for eye tracking are electrooculography (EOG), in which electrodes are attached to the skin to measure the variation of the electric field of the eyeball; infrared oculography (IROG), in which the amount of infrared light reflected by the eye indicates the movement of the eyeball; video oculography (VOG), in which a video camera is used to estimate the direction of the eyeball; and the scleral search coil method, in which a coil is attached to the eye and subjected to an external magnetic field, whereby voltage is induced in the coil upon the movement of the eyeball. ${ }^{[2,9]}$ However, these methods are uncomfortable, expensive, invasive, require wire connections, or need the eyes to be wide open. Furthermore, none of these methods can be used in everyday life. Schwarz et al. ${ }^{[10]}$ succeeded in tracking the eye movements of freely behaving animals using a compact device, namely implanting a magnet inside the eye, but this is not a practical solution for wearable devices. 


\section{WILEY-VCH}

Table S1. Advantageous and disadvantageous of existing eye-tracking methods ${ }^{[11]}$

\begin{tabular}{|c|c|c|}
\hline Technique & Advantage & Disadvantage \\
\hline $\begin{array}{l}\text { Electro-oculography } \\
\text { (EOG) }\end{array}$ & $\begin{array}{l}\text { Cheap, easy and non- } \\
\text { invasive }\end{array}$ & $\begin{array}{l}\text { - Sensitive to variation in light } \\
\text { intensity } \\
\text { - Requires physical wires to be } \\
\text { attached to the patient }\end{array}$ \\
\hline $\begin{array}{l}\text { Infrared oculography } \\
\text { (IROG) }\end{array}$ & $\begin{array}{l}\text { Good resolution for } \\
\text { horizontal eye-movement }\end{array}$ & $\begin{array}{l}\text { - Obstructs the patient view due } \\
\text { to the existence of the } \\
\text { recording device within the } \\
\text { visual field of the patient } \\
\text { - Recording with corrective } \\
\text { spectacles, contact lenses or } \\
\text { with closed eyes is not } \\
\text { possible }\end{array}$ \\
\hline $\begin{array}{l}\text { Scleral search coil } \\
\text { method }\end{array}$ & $\begin{array}{l}\text { precise three-dimensional } \\
\text { recording of eye } \\
\text { movements }\end{array}$ & $\begin{array}{l}\text { - Invasive } \\
\text { - Requires special technical } \\
\text { knowledge } \\
\text { - Recording duration is limited } \\
\text { to an hour at maximum }\end{array}$ \\
\hline $\begin{array}{l}\text { Video oculography } \\
\text { (VOG) }\end{array}$ & $\begin{array}{l}\text { Non-invasive and provides } \\
\text { quantitative eye position } \\
\text { records in two or three } \\
\text { dimensions }\end{array}$ & $\begin{array}{l}\text { - Recording with closed eyes is } \\
\text { not possible }\end{array}$ \\
\hline $\begin{array}{l}\text { Implantable magnet } \\
{[10]}\end{array}$ & $\begin{array}{l}\text { Unobtrusive system and } \\
\text { ability to track eye } \\
\text { movement of free behaving } \\
\text { animals }\end{array}$ & $\begin{array}{l}\text { - Invasive and requires surgical } \\
\text { insertion of a magnet inside } \\
\text { the eyeball }\end{array}$ \\
\hline
\end{tabular}




\section{WILEY-VCH}

\section{Supplementary Information-4: Assessment the comfort level of wearing the magnetic}

skin

A survey was conducted to evaluate the comfort level and the impact of having the magnetic skin attached to the eyelid. The survey was regulated by the Saudi National Committee of Bio-Ethics (NCBE) and the Institutional Biosafety and Bioethics Committee (IBEC) at King Abdullah University of Science and Technology. The survey revolved around the three questionnaire points indicated in Table S2, with a sample size of 30 volunteers (10 females and 20 males aged from 17 to 36). The survey was carried out by attaching a black 10 x $2 \times 0.7 \mathrm{~mm}^{3}$ magnetic skin to the eyelid using Vaseline ${ }^{\circledR}$ (a biocompatible and clinically proven petroleum jelly). The volunteers were asked to keep the magnetic skin attached to the eyelid for at least 30 minutes before proceeding with the survey. Supplementary Table 1 presents the results, which indicate that the magnetic skin is highly comfortable (i.e., physically and emotionally) to wear. With a confidence level exceeding $95 \%(\mathrm{p}<0.05$, student's t-test), the discomfort level for all the different criteria in Supplementary Table 1 is below 1.2, with 0 meaning that the volunteer was not affected by the magnetic skin at all and 5 meaning it had a strong effect. The small percentage of participants with an answer $\geq 2$ for the discomfort level complained about having the Vaseline ${ }^{\circledR}$ on the eyelid, suggesting that the use of another, less viscous material could remedy this issue.

Table S2. Evaluating the comfort of wearing the magnetic skin on the eyelid

\begin{tabular}{|c|c|c|c|c|c|c|c|c|}
\hline \multirow[b]{2}{*}{ Survey question } & \multicolumn{6}{|c|}{ Discomfort level } & \multirow[b]{2}{*}{$\begin{array}{c}\text { Sample } \\
\text { mean } \\
(\mu)\end{array}$} & \multirow[b]{2}{*}{$\begin{array}{l}\text { Standard } \\
\text { deviation } \\
\quad(\sigma)\end{array}$} \\
\hline & $\begin{array}{c}0 \\
\text { No } \\
\text { effect }\end{array}$ & 1 & 2 & 3 & 4 & $\begin{array}{c}5 \\
\text { Strong } \\
\text { effect }\end{array}$ & & \\
\hline $\begin{array}{l}\text { 1. The comfort level (i.e., is } \\
\text { magnetic skin annoying to wear?) }\end{array}$ & $37 \%$ & $43 \%$ & $17 \%$ & $3 \%$ & $0 \%$ & $0 \%$ & 0.87 & 0.82 \\
\hline $\begin{array}{l}\text { 2. The physical effects (i.e., pain, } \\
\text { irritation, } \ldots \text { etc.) }\end{array}$ & $73 \%$ & $27 \%$ & $0 \%$ & $0 \%$ & $0 \%$ & $0 \%$ & 0.27 & 0.45 \\
\hline $\begin{array}{l}\text { 3. The emotional comfort (i.e., the } \\
\text { appearance) }\end{array}$ & $73 \%$ & $3 \%$ & $10 \%$ & $10 \%$ & $3 \%$ & $0 \%$ & 0.67 & 1.21 \\
\hline
\end{tabular}




\section{WILEY-VCH}

Supplementary Information-5: Electrical interface of the touchless light switch

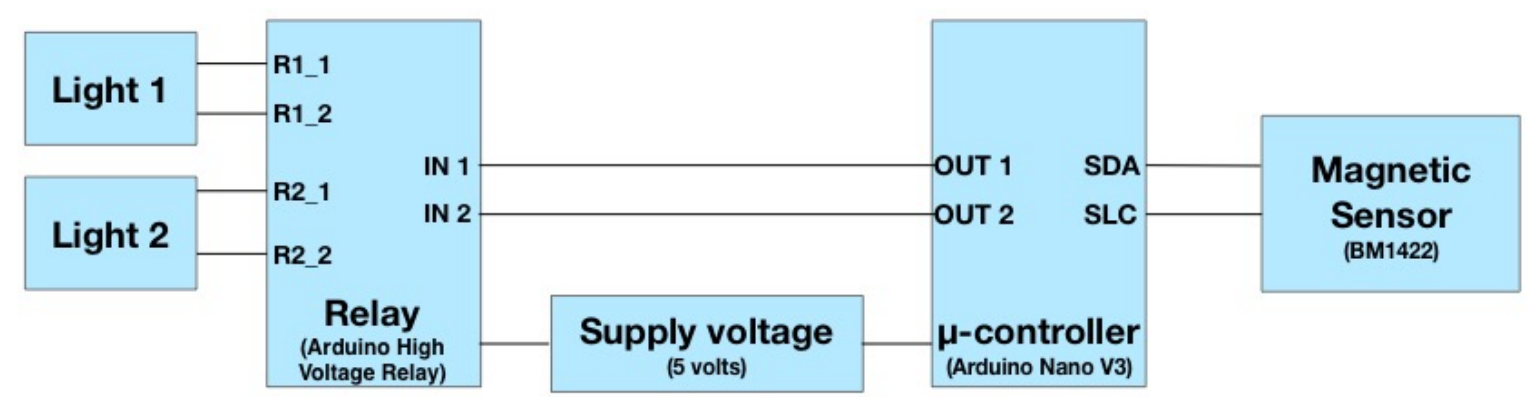

Figure S4. Functional block diagram of the electrical interface for the touchless control lightswitch. 


\section{WILEY-VCH}

References

[1] Skotte, J.; Nøjgaard, J.; Jørgensen, L.; Christensen, K.; Sjøgaard, G., European journal of applied physiology 2007, 99 (2), 113-119.

[2] Duchowski, A. T., Theory and practice 2007, 328.

[3] Majaranta, P.; Bulling, A., Eye tracking and eye-based human-computer interaction. In Advances in physiological computing, Springer: 2014; pp 39-65.

[4] Horowitz, B. T. Eye-tracking technology allows paralyzed people to control PCs, tablets. https://blog.dell.com/en-us/eye-tracking-technology-allows-paralyzed-people-tocontrol-pcs-tablets/ (accessed February 14).

[5] Corcoran, P. M.; Nanu, F.; Petrescu, S.; Bigioi, P., IEEE Transactions on Consumer Electronics 2012, 58 (2).

[6] Takahashi, K.; Atsumi, Y., Sleep 1997, 20 (9), 743-752.

[7] Frueh, B. R., Ophthalmology 1980, 87 (10), 1019-1021; Azri, M.; Young, S.; Lin, H.; Tan, C.; Yang, Z. In Diagnosis of Ocular Myasthenia Gravis by means of tracking eye parameters, Engineering in Medicine and Biology Society (EMBC), 2014 36th Annual International Conference of the IEEE, IEEE: 2014; pp 1460-1464.

[8] Smith, P.; Shah, M.; da Vitoria Lobo, N. In Monitoring head/eye motion for driver alertness with one camera, icpr, IEEE: 2000; p 4636.

[9] Singh, H.; Singh, J., International Journal of Scientific and Research Publications 2012, 2 (9), 1-9.

[10] Schwarz, J. S.; Sridharan, D.; Knudsen, E. I., Frontiers in systems neuroscience 2013, 7, 91 .

[11] Heide, W.; Koenig, E.; Trillenberg, P.; Kömpf, D.; Zee, D., Electroencephalogr Clin Neurophysiol Suppl 1999, 52, 223-240. 